\title{
Xây dựng khung đánh giá năng lực giảng dạy của sinh viên chuyên ngành phương pháp giảng dạy tiếng Anh
}

\section{The development of a framework for assessing student teachers' English teaching competency}

\author{
Trần Quốc Thao ${ }^{1 *}$ \\ ${ }^{1}$ Trường Đại học Công nghệ Thành phố Hồ Chí Minh (HUTECH), Việt Nam \\ "Tác giả liên hệ, Email: tq.thao@ hutech.edu.vn
}

\begin{abstract}
THÔNG TIN
TÓM TẮT

DOI: $10.46223 / \mathrm{HCMCOUJS.}$ Soci.vi.15.1.593.2020

Ngày nhận: 07/04/2020

Ngày nhận lại: 06/05/2020

Duyệt đăng: 02/06/2020

Tì khóa:

khung đánh giá, năng lực giảng dạy, phương pháp giảng dạy, sinh viên tiếng Anh

Xây dựng khung đánh giá năng lực giảng dạy (NLGD) của sinh viên (SV) là một trong những nhiệm vụ quan trọng trong việc đảm bảo chất lượng đào tạo giáo viên nói chung và giáo viên tiếng Anh nói riêng. Bài báo này trình bày kết quả của việc xây dựng khung đánh giá NLGD của SV chuyên ngành phương pháp giảng dạy (PPGD) tiếng Anh tại một Trường đại học ở Thành phố Hồ Chí Minh. Ba bước (Analyze: phân tích; Design: thiết kế; Develop: phát triển) của mô hình ADDIE được áp dụng để xây dựng khung đánh giá NLGD tiếng Anh. Để thu thập dữ liệu, 93 SV chuyên ngành PPGD tiếng Anh tham gia trả bảng câu hỏi, và ba chuyên gia tham gia thẩm định khung đánh giá NLGD tiếng Anh. Kết quả của nghiên cứu là khung đánh giá NLGD của $S V$ chuyên ngành PPGD tiếng Anh. Khung đánh giá này có thể hữu dụng và phù hợp cho việc đào tạo chuyên ngành PPGD tiếng Anh tại nơi nghiên cứu.
\end{abstract}

Keywords:

framework, English teaching competency, TESOL methodology, student teacher

\section{ABSTRACT}

Developing a framework for the assessment of teaching competency of student teachers is one of the important missions for ensuring the quality of student teachers in general and student teachers of English in specific. This paper presents how a framework for the assessment of teaching competency of student teachers (FATCST) was developed. Three steps (analyze, design and develop) of the ADDIE model were applied for the development of FATCST. Ninety-three student teachers majoring in TESOL methodology from a Ho Chi Minh-based higher institution joined the study by answering a questionnaire and three experts took part in evaluating the FATCST. The study finally came up with a complete FATCST which may be useful and appropriate for the assessment of teaching competency of student teachers at the institution. 


\section{1. Đặt vấn đề}

Việc xây dựng khung đánh giá Năng Lực Giảng Dạy (NLGD) là một trong những công việc quan trọng giúp nâng cao chất lượng giảng dạy của Giáo Viên $(\mathrm{GV})$. Alqiawi và Ezzeldin (2015) đã khẳng định NLGD của GV có thể dùng để định hướng và xác định phẩm chất và đặc điểm cá nhân của $\mathrm{GV}$ dạy giỏi. Tương tự, trong lĩnh vực đào tạo $\mathrm{GV}$, xây dựng khung đánh giá NLGD là một trong những yếu tố quan trọng giúp đảm bảo chất lượng đào tạo. Bhargava và Pathy (2011) chỉ ra rằng $\mathrm{GV}$ trong các lĩnh vực giảng dạy cụ thể cần giảng dạy dựa vào các tiêu chí NLGD đã được đề ra trong lĩnh vực của mình và các chương trình đào tạo $\mathrm{GV}$ cũng nên chú trọng đến các tiêu chí NLGD đó để giúp cho người dạy và người học thực hiện đúng các vai trò của mình và đảm bảo chất lượng dạy và học. Do đó, xây dựng khung đánh giá NLGD nói chung và NLGD tiếng Anh nói riêng nên đặt thành một trong những ưu tiên hàng đầu đối với các cơ sở đào tạo $\mathrm{GV}$.

Ở Việt Nam, tiếng Anh là ngoại ngữ bắt buộc được dạy ở các cấp bậc khác nhau, nên việc đào tạo $\mathrm{GV}$ tiếng Anh đã và đang là một trong những yêu cầu cần thiết đối với các cơ sở đào tạo $\mathrm{GV}$, và việc đào tạo $\mathrm{GV}$ tiếng Anh theo chuẩn nghề nghiệp là một trong những yêu cầu hàng đầu trong công việc đào tạo $\mathrm{GV}$. Trước tình hình nhu cầu cần GV tiếng Anh ngày càng tăng, nhiều cơ sở giáo dục bắt đầu triển khai đào tạo GV tiếng Anh (Tran, 2020). Trên cơ sở các quy định và hướng dẫn về việc đánh giá NLGD của GV các cấp do Bộ Giáo Dục và Đào Tạo (BGD \& ĐT) Việt Nam đã ban hành, nhiều trường đào tạo GV xây dựng khung đánh giá NLGD nhằm đảm bảo chất lượng đào tạo GV theo chuẩn của BGD \& ĐT đã đề ra. Do đó, bài báo này trình bày khung đánh giá NLGD tiếng Anh của $\mathrm{SV}$ chuyên ngành Phương Pháp Giảng Dạy (PPGD) tiếng Anh tại một trường đại học tại Thành phố Hồ Chí Minh. Trong phạm vi nghiên cứu này, câu hỏi nghiên cứu được đặt ra như sau:

- Khung đánh giá NLGD tiếng Anh của SV chuyên ngành PPGD tiếng Anh được xây dựng tại 1 trường đại học tại Thành phố Hồ Chí Minh như thế nào?

\section{Cơ sở lý luận}

Thuật ngữ năng lực ở góc độ chung, theo Deakin (2008) thì được mô tả là sự kết hợp phức tạp của kiến thức, kỹ năng, sự hiểu biết, các giá trị, thái độ và đam mê để giúp con người thực hiện hành vi hiệu quả. Khi xét ở góc độ giảng dạy, thì NLGD bao gồm các yếu tố như kiến thức, kỹ năng thực tế, động lực, niềm tin, định hướng giá trị và tình cảm (Rychen \& Salganik, 2003). Năng lực giúp cho giáo viên đáp ứng được các yêu cầu phức tạp, và giúp họ thực hiện một cách chuyên nghiệp và phù hợp trong tình huống cụ thể (Koster \& Dengirnk, 2008).

Đặc điểm của năng lực như sau:

- Năng lực bao gồm một hoặc nhiều kỹ năng giúp cho việc duy trì năng lực đó;

- Năng lực có tính liên kết của 3 lĩnh vực thành phần (kiến thức, kỹ năng, và thái độ) được dùng để xem xét khi một hành động được đánh giá;

- Năng lực có thể thấy được và thể hiện;

- Năng lực có thể được đánh giá.

(Barman \& Paramanik, 2019, p. 479) 
NLGD khác với năng lực giáo viên. NLGD thiên về vai trò của giáo viên trong lớp học, trực tiếp liên quan đến giảng dạy (Hagger \& McIntyre, 2006), còn năng lực giáo viên với hàm ý rộng hơn về năng lực nghề nghiệp ở nhiều góc độ - cá nhân, nhà trường, cộng đồng, và mạng lưới nghề nghiệp. Tuy nhiên, hai khái niệm này đôi khi được dùng để biểu thị cho nhau.

Trong phạm vi nghiên cứu này, NLGD của giáo viên được hiểu là khả năng giảng dạy bao gồm thái độ, kiến thức và kỹ năng nhằm giúp cho họ có đủ năng lực truyền đạt kiến thức và giải quyết các tình huống sư phạm cụ thể trong lớp học.

\section{Thái độ}

Thái độ liên quan đến cam kết, tự tin, tin tưởng và tôn trọng trong giảng dạy, và nó có thể là tích cực hoặc tiêu cực. Thái độ là cầu nối giữa kiến thức và kỹ năng nhằm giúp giáo viên thực hiện giảng dạy hướng tới mục đích giáo dục chung và tối đa hóa tiềm năng học tập của người học (Council of Europe, 2008; Feiman-Nemser, 2008).

\section{Kiến thức}

Giáo viên cần có kiến thức chuyên môn sâu rộng cả về phương pháp và chuyên môn (Krauss et al., 2008; Shulman, 1987) để giảng dạy hiệu quả trong các môi trường giảng dạy khác nhau (McDiarmid \& Clevenger-Bright, 2008). Để thực hiện được nhiệm vụ đó, giáo viên cần kiến thức về chương trình học, quản lý lớp, phương pháp dạy học, lý thuyết về giáo dục và đánh giá người học (Darling-Hammong \& Branford, 2005) và hiểu biết về công nghệ thông tin (CNTT) có thể áp dụng trong giảng dạy (Mishra \& Koehler, 2006).

\section{Kỹ năng}

NLGD của giáo viên được thể hiện qua khả năng linh hoạt giảng dạy nhằm đáp ứng năng lực học tập của người học (Hatano \& Oura, 2003; Vogt \& Rogalla, 2009). Kỹ năng giảng dạy còn được thể hiện qua việc phân bố chương trình học, quản lý lớp học, sử dụng chiến lược dạy học, kiểm tra và đánh giá người học (Scheerens, Luyten, Steen, \& Luyten-de Thouars, 2007). Ngoài ra, kỹ năng giảng dạy còn thể hiện qua khả năng đánh giá có hệ thống dựa trên lý thuyết, nghiên cứu, kinh nghiệm nghề nghiệp và minh chứng nhằm mục đích cải thiện (Hagger \& McIntyre, 2006).

Tóm lại, như đã đề cập ở trên, các yếu tố cấu thành NLGD gồm ba thành tố chủ yếu là thái độ, kiến thức và kỹ năng. Ba thành tố này có mối quan hệ mật thiết, bổ trợ cho nhau. Trong ngữ cảnh xem xét NLGD tiếng Anh của một người đang được đào tạo để trở thành giáo viên, NLGD tiếng Anh bao gồm bốn thành tố cơ bản thái độ, kiến thức, kỹ năng, và năng lực ngôn ngữ tiếng Anh, và chúng được xem xét ở góc độ hẹp hơn.

Tính tới nay, có nhiều khung đánh giá NLGD tiếng Anh được đề xuất; tuy nhiên, trong phạm vi nghiên cứu này khung đánh giá NLGD dành cho giáo viên tiếng Anh được đề cập. Năm 2008, tại Anh Quốc khung đánh giá NLGD tiếng Anh học thuật BALEAP (BALEAP Competency Framework for Teachers of English for Academic Purposes) được xây dựng và giới thiệu nhằm hỗ trợ phát triển nghề nghiệp cho giáo viên giảng dạy tiếng Anh học thuật (BALEAP, 2008). Khung đánh giá NLGD tiếng Anh học thuật BALEAP không có tham chiếu về mức độ NLGD của giáo viên, nhưng dựa vào khung lý thuyết. Khung này gồm 4 nhóm năng 
lực chính: (1) Năng lực liên quan đến hoạt động học thuật; (2) Năng lực liên quan đến SV tiếng Anh học thuật; (3) Năng lực liên quan đến phát triển khung chương trình; (4) Năng lực liên quan đến giảng dạy.

Năm 2010, Trung tâm ngôn ngữ ứng dụng tại Washington, Mỹ giới thiệu khung phát triển nghề nghiệp CAELA (CAELA Framework for Professional Development) nhằm hỗ trợ cho chương trình đào tạo giáo viên và phát triển năng lực người học thông qua chương trình phát triển nghề nghiệp bền vững, liên kết và hệ thống. Tuy nhiên, khung phát triển nghề nghiệp CAELA không có tham chiếu về mức độ NLGD của giáo viên (CAELA, 2010). Cũng tại Anh Quốc, khung đánh giá NLGD của GV tiếng Anh do Hội đồng Anh (British Council CPD Framework for Teachers of English) phát triển năm 2011 nhằm hỗ trợ giáo viên phát triển nghề nghiệp và chọn hoạt động phát triển nghề nghiệp phù hợp nhất cho bản thân (British Council, 2011). Giai đoạn đầu, khung đánh giá NLGD này đưa ra 6 mức độ đánh giá NLGD cụ thể. Đến năm 2012, khung đánh NLGD của giáo viên tiếng Anh được bổ sung và hoàn thiện kèm theo hướng dẫn để giúp giáo viên tự xác định mức độ của mình (British council, 2012). Trong 2015, Hội đồng Anh tiếp tục điều chỉnh và đưa ra 4 giai đoạn phát triển (1. Nhận thức -> 2. Hiểu biết -> 3. Tham gia -> 4. Hội nhập) và 12 nội dung hoạt động nghề nghiệp: (1) Soạn giáo trình và bài giảng; (2) Hiểu về người học; (3) Quản lý bài giảng; (4) Hiểu biết về môn học; (5) Quản lý tài nguyên dạy-học; (6) Đánh giá việc học; (7) Ứng dụng CNTT vào giảng dạy; (8) Tự phát triển nghề nghiệp; (9) Sử dụng phương pháp đồng bộ; (10) Sử dụng phương pháp đa ngôn ngữ; (11) Phát triển kỹ năng của thế kỹ 21; (12) Hiểu về phương pháp và chính sách giáo dục.

Năm 2013 tại Anh, các nhà nghiên cứu đã xây dựng khung đào tạo và phát triển giáo viên ngôn ngữ EAQUALS (EAQUALS Framework for Language Teacher Training and Development) nhằm giúp cho giáo viên, nhà đào tạo giáo viên và nhà quản lý đánh giá NLGD của giáo viên (EAQUALS, 2013). Khung đào tạo và phát triển giáo viên ngôn ngữ EAQUALS mô tả về phát triển năng lực nghề nghiệp theo 3 yếu tố: thái độ, kiến thức và kỹ năng của giáo viên (EAQUALS, 2013). Khung EAQUALS bao gồm 5 tiêu chuẩn: (1) Lập kế hoạch day và học; (2) Dạy và hỗ trợ học; (3) Đánh giá học; (4) Ngôn ngữ, giao tiếp và văn hóa; (5) Nghề nghiệp giáo viên.

Khung GDTA của Cambridge (Cambridge English Teaching Framework: CETF) (UCLES, 2015) được xây dựng để tóm lược kiến thức và kỹ năng cần thiết để giảng dạy hiệu quả trong các giai đoạn và hoàn cảnh khác nhau. Mục đích của CETF nhằm hỗ trợ giáo viên xác định năng lực nghề nghiệp của họ ở mức độ nào và giúp giáo viên và lãnh đạo định hướng hoạt động để đạt chuẩn nghề nghiệp. Khung CETF gồm 5 tiêu chuẩn (1) Học và người học, (2) Dạy, học và đánh giá, (3) Năng lực ngôn ngữ, (4) Kiến thức và nhận thức về ngôn ngữ, và (5) Giá trị và phát triển nghề nghiệp, và các tiêu chuẩn này gồm 36 tiêu chí dùng để đánh giá NLGD của giáo viên theo 4 mức độ: căn bản, phát triển, thành thạo và chuyên gia.

Ở Việt Nam, Bộ Giáo dục và Đào tạo ban hành khung đánh giá năng lực ngoại ngữ 6 bậc (BGDĐT, 2014) nhằm mục đích đánh giá năng lực ngoại ngữ của giáo viên. Khung năng lực này chia làm 3 cấp (Sơ cấp, Trung cấp và Cao cấp) theo 6 bậc (từ Bậc 1 đến Bậc 6 và tương thích với các bậc từ $\mathrm{A} 1$ đến $\mathrm{C} 2$ trong $\mathrm{CEFR}$ ). Tuy nhiên, khung đánh giá này chỉ hướng tới đánh giá năng lực ngoại ngữ chứ không đánh giá NLGD của giáo viên ngoại ngữ. Ngoài ra, 
năm 2012, nhà nghiên cứu Duong, Pham, và Thai xây dựng khung năng lực đánh giá cho giáo viên và giáo sinh dạy tiếng Anh. Trên cơ sở phân tích các khung đánh giá đã có trước, nhóm tác giả đã đề xuất khung năng lực đánh giá gồm 4 cấp độ bao gồm nhận thức, xử lý, tiếp thu, và thể hiện kiến thức qua ngôn ngữ. Tuy nhiên, khung năng lực đánh giá này chỉ mới dừng lại ở mức độ lý thuyết, chưa được kiểm chứng qua thực tiễn. Năm 2016, nhà nghiên cứu Pham và Ta đã phát triển khung đào tạo giáo viên dạy tiếng Anh chuyên ngành (ESP). Trên cơ sở tổng hợp lý thuyết và phân tích tình hình thực tiễn của việc đào tạo giáo viên dạy tiếng Anh chuyên ngành, hai nhà nghiên cứu đã xây dựng khung đào tạo giáo viên dạy tiếng Anh chuyên ngành ở Việt Nam. Khung đào tạo này gồm 2 thành phần chính: Phương pháp giảng dạy tiếng Anh chuyên ngành và thụ đắc về tiếng Anh chuyên ngành. Khung đào tạo này cũng như khung năng lực đánh giá của nhà Duong và cộng sự (2012) là chỉ dừng lại ở mức độ lý thuyết, chưa được kiểm chứng qua thực tiễn. Năm 2017, các nhà nghiên cứu Bui, Nguyen, Dao, và Hoan đã xây dựng tiêu chuẩn nghề nghiệp cho giáo viên Việt Nam các môn học nói chung. Họ dựa trên kinh nghiệm của

Singapore và phân tích đánh giá tiêu chuẩn nghề; từ đó họ đã đưa ra các đề xuất để xây dựng các tiêu chuẩn nghề nghiệp cho giáo viên Việt Nam. Cũng như các nghiên cứu trước, các tiêu chuẩn nghề nghiệp cho giáo viên cũng chỉ ở mức lý thuyết mà các tác giả chưa thẩm định được các tiêu chuẩn qua thực tế.

\section{Phương pháp nghiên cứu}

\subsection{Thiết kế nghiên cúu}

Nghiên cứu này được thực hiện trên cơ sở tổng hợp và phân tích các cơ sở lý thuyết và ý kiến khảo sát từ SV chuyên ngành PPGD tiếng Anh để xây dựng khung đánh giá NLGD tiếng Anh. Để xây dựng khung đánh giá NLGD tiếng Anh hoàn chỉnh, ba bước của mô hình ADDIE (Branch, 2009) (Analyze: phân tích; Design: thiết kế; Develop: phát triển) được áp dụng.

\section{Bước 1: Phân tích (Analyze)}

1. Xác định nhu cầu và mục đích

2. Phân tích môi trường học

3. Phân tích đặc điểm người học

\section{Bước 2: Thiết kế (Design)}

4. Xác định mục tiêu khung đánh giá NLGD

5. Xác định nội dung khung đánh giá NLGD

\section{Bước 3: Phát triển (Develop)}

6. Phát triển khung đánh giá NLGD

7. Thẩm định khung đánh giá NLGD

8. Điều chỉnh khung đánh giá NLGD 


\subsection{Nơi nghiên cúu}

Nghiên cứu này được thực hiện tại một trường đại học ở Thành phố Hồ Chí Minh. Trường này là một trong những trường đại học đa ngành tại Việt Nam. Trong đó, Khoa tiếng Anh đào tạo ba chuyên ngành: tiếng Anh biên phiên dịch, tiếng Anh thương mại, và PPGD tiếng Anh. Chuyên ngành PPGD tiếng Anh là chuyên ngành mới đối với Khoa tiếng Anh. Các môn thuộc chuyên ngành PPGD tiếng Anh gồm có PPGD tiếng Anh 1 (3 tín chỉ), PPGD tiếng Anh 2 (3 tín chỉ), Quản lý lớp học (3 tín chỉ), Ngôn ngữ đối chiếu và giảng dạy ngôn ngữ (3 tín chỉ), và Thực tập sư phạm (3 tín chỉ). Môn PPGD tiếng Anh 1 và 2 dạy về các lý thuyết xoay quanh các vấn đề giảng dạy tiếng Anh, các phương pháp và kỹ thuật giảng dạy tiếng Anh và thực hành tập giảng; môn Quản lý lớp học tập trung giúp người học về kỹ năng sử dụng ngôn ngữ và tổ chức quản lý lớp học; môn Ngôn ngữ đối chiếu và giảng dạy ngôn ngữ giúp cho người học hiểu rõ những điểm tương đồng và dị biệt giữa ngôn ngữ Việt và Anh xét về cấu trúc nội dung và biểu đạt để ứng dụng thực tế vào việc giảng dạy tiếng Anh; môn Thực tập sư phạm giúp cho người học đi thực tế thực tập các công việc của một $\mathrm{GV}$ thực thụ.

\section{3. Đối tuọng khảo sát}

Đối tượng khảo sát gồm SV chuyên ngành PPGD tiếng Anh và chuyên gia. Theo Bảng 1, số người tham gia khảo sát là $93 \mathrm{SV}$ (gồm 21 nam; 72 nữ), và số $\mathrm{SV}$ này được chọn có chủ đích vì họ đã đăng ký học chuyên ngành giảng dạy tiếng Anh. Độ tuổi từ 21-30 chiếm 98,9\% (92 SV), và chỉ có $1 \mathrm{SV}$ dưới 20 tuổi $(1,1 \%)$. Về thời gian đã học TA, có $75 \mathrm{SV}(80,6 \%)$ trên tổng số $93 \mathrm{SV}$ đã học TA trên 10 năm, kế tiếp là $17 \mathrm{SV}$ (18,3\%) đã học TA từ 5-10 năm; chỉ duy nhất có $1 \mathrm{SV}(1,1 \%)$ đã học TA dưới 5 năm. Trong tổng số $93 \mathrm{SV}$ thì có $52 \mathrm{SV}$ có kinh nghiệm giảng dạy như trợ giảng $(22,6 \%)$, gia sư $(26 \%)$ và GV chính thức $(6,5 \%)$, và số SV có kinh nghiệm giảng dạy dưới 1 năm là $23(24,8 \%)$, từ $1-2$ năm là $20(21,5 \%)$ và trên 2 năm là 20 (21,5\%). Ngoài ra, có $26 \mathrm{SV}(28 \%)$ đã từng học khóa học về PPGD tiếng Anh.

\section{Bảng 1}

Thông tin cá nhân của SV tham gia khảo sát

\begin{tabular}{|c|c|c|c|c|}
\hline \multirow{2}{*}{ STT } & \multirow{2}{*}{ Thông tin } & & \multicolumn{2}{|c|}{$n=93$} \\
\hline & & & $\mathbf{F}$ & $\%$ \\
\hline \multirow{2}{*}{1} & \multirow{2}{*}{ Giới tính } & Nam & 21 & 22,6 \\
\hline & & Nữ & 72 & 77,4 \\
\hline \multirow{3}{*}{2} & \multirow{3}{*}{ Độ tuổi } & dưới 20 & 1 & 1,1 \\
\hline & & $21-30$ & 92 & 98,9 \\
\hline & & trên 30 & 0 & $0,0 \mathrm{~s}$ \\
\hline \multirow{3}{*}{3} & \multirow{3}{*}{ Đã học tiếng Anh } & dưới 5 năm & 1 & 1,1 \\
\hline & & $5-10$ & 17 & 18,3 \\
\hline & & trên 10 & 75 & 80,6 \\
\hline \multirow{3}{*}{4} & \multirow{3}{*}{$\begin{array}{l}\text { Lý do chọ́n chuyên ngành } \\
\text { PPGD tiếng Anh }\end{array}$} & Đam mê & 52 & 55,9 \\
\hline & & Định hướng từ người thân & 30 & 32,3 \\
\hline & & khác & 11 & 11,8 \\
\hline
\end{tabular}




\begin{tabular}{|c|c|c|c|c|}
\hline \multirow{2}{*}{ STT } & \multirow{2}{*}{ Thông tin } & & \multicolumn{2}{|c|}{$n=93$} \\
\hline & & & $\mathbf{F}$ & $\%$ \\
\hline \multirow{4}{*}{5} & \multirow{4}{*}{$\begin{array}{l}\text { Kinh nghiệm giảng dạy tiếng } \\
\text { Anh }\end{array}$} & Trợ giảng & 21 & 22,6 \\
\hline & & Gia sư & 25 & 26,9 \\
\hline & & GV chính thức & 6 & 6,5 \\
\hline & & Chưa & 41 & 44,1 \\
\hline \multirow{3}{*}{6} & \multirow{3}{*}{ Kinh nghiệm dạy được } & dưới 1 năm & 23 & 24,8 \\
\hline & & 1-2 năm & 20 & 21,4 \\
\hline & & Trên 2 năm & 9 & 9,7 \\
\hline \multirow{2}{*}{7} & \multirow{2}{*}{$\begin{array}{l}\text { Đã từng học khóa học PPGD } \\
\text { tiếng Anh }\end{array}$} & Có & 26 & 28,0 \\
\hline & & Chưa & 67 & 72,0 \\
\hline
\end{tabular}

Trong đó: n: số lượng mẫu; F: tần suất; \%: phần trăm

Nguồn: Kết quả phân tích dữ liệu của nhóm nghiên cứu

Để thu thập thông tin đánh giá khung đánh giá NLGD tiếng Anh, ba chuyên gia được mời có chủ đích để đánh giá. Theo thông tin ở Bảng 2 cho thấy, ba chuyên gia ( 1 nam; 2 nữ) có độ tuổi từ 30 trở lên, và đều có trình độ chuyên môn là tiến sĩ và đều dạy học ở cấp đại học, Trong ba chuyên gia thì có một người đã kinh nghiệm giảng dạy trên 20 năm, hai người còn lại đã giảng dạy từ 16 năm trở lên.

\section{Bảng 2}

Thông tin cá nhân của chuyên gia đánh giá khung đánh giá NLGD tiếng Anh

\begin{tabular}{|c|c|c|c|}
\hline STT & Thông tin & & $\mathbf{n}=\mathbf{3}$ \\
\hline \multirow{2}{*}{1} & \multirow{2}{*}{ Giới tính } & Nam & 1 \\
\hline & & Nữ & 2 \\
\hline \multirow{4}{*}{2} & \multirow{4}{*}{ Độ tuổi } & Dưới 30 & 0 \\
\hline & & $30-40$ & 1 \\
\hline & & $41-50$ & 1 \\
\hline & & Trên 50 & 1 \\
\hline \multirow{3}{*}{3} & \multirow{3}{*}{ Trình độ chuyên môn } & Thạc sĩ & 0 \\
\hline & & Tiến sĩ & 3 \\
\hline & & Khác & 0 \\
\hline \multirow{3}{*}{4} & \multirow{3}{*}{ Cấp dạy } & Phổ thông & 0 \\
\hline & & Đại Học & 3 \\
\hline & & Tự do & 0 \\
\hline \multirow{4}{*}{5} & \multirow{4}{*}{ Năm giảng dạy } & Dưới 10 & 0 \\
\hline & & $11-15$ & 0 \\
\hline & & $16-20$ & 2 \\
\hline & & Trên 20 & 1 \\
\hline
\end{tabular}

Trong đó: n: số lượng mẫu

Nguồn: Kết quả phân tích dữ liệu của nhóm nghiên cứu 


\subsection{Công cụ nghiên cú́u}

Hai công cụ nghiên cứu được sử dụng để thu thập dữ liệu là bảng câu hỏi cho $\mathrm{SV}$ trước khóa học và phiếu đánh giá khung NLGD dành cho chuyên gia.

Bảng câu câu hỏi cho SV trước khóa học

Bảng câu câu hỏi cho $\mathrm{SV}$ được xây dựng trên cơ sở nhằm mục đích tìm hiểu các thông tin của SV trước khóa học PPGD tiếng Anh. Nội dung bảng câu hỏi được xây dựng dựa trên cơ sở lý thuyết và nó gồm có hai phần: Phần $\mathrm{A}$ hỏi về thông tin cá nhân; phần $\mathrm{B}$ là nội dung câu hỏi. Phần câu hỏi gồm có 26 câu lựa chọn sử dụng thang đo có năm mức độ từ Hoàn toàn không đồng ý tới hoàn toàn đồng ý, và các câu hỏi này được chia thành ba nhóm câu hỏi: Thái độ, kiến thức và kỹ năng về giảng dạy tiếng Anh của SV trước khóa học PPGD tiếng Anh. Nhóm câu hỏi về Thái độ về GDTA gồm bảy câu hỏi (câu 1 đến câu 7) với Cronbach $\alpha$ là 0,82 ; nhóm câu hỏi về Kiến thức về GDTA gồm chín câu hỏi (câu 8 đến câu 16) với Cronbach $\alpha$ là 0,95 ; nhóm câu hỏi về về GDTA gồm mười câu hỏi (câu 17 đến câu 26) với Cronbach $\alpha$ là 0,96 . Tổng Cronbach $\alpha$ của bảng câu hỏi là 0,96 . Điều này có nghĩa là bảng câu hỏi có độ tin cậy cao.

Phiếu đánh giá khung đánh giá NLGD tiếng Anh dành cho chuyên gia

Nội dung phiếu dánh giá bao gồm hai phần: thông tin cá nhân và nội dung. Phần nội dung bao gồm năm câu hỏi về các thông tin cá nhân của chuyên gia. Phần nội dung gồm 75 câu hỏi được chia thành ba phần nhỏ:

- Phần I: Mục tiêu của của khung đánh giá NLGD tiếng Anh. Nó gồm 16 câu hỏi về mục tiêu và các tiêu chuẩn của khung đánh giá NLGD tiếng Anh và chúng được sử dụng thang đo năm bậc từ Hoàn toàn không đồng ý tới Hoàn toàn đồng ý. Ngoài ra, phần này còn có một câu hỏi phụ hỏi về ý kiến góp ý cho nội dung của mục tiêu và tiêu chuẩn của khung đánh giá NLGD tiếng Anh;

- Phần II: Nội dung chi tiết của khung đánh giá NLGD tiếng Anh. Nó gồm 33 câu hỏi về nội dung các tiêu chuẩn và tiêu chí của khung đánh giá NLGD tiếng Anh, và chúng được sử dụng thang đo năm bậc từ Hoàn toàn không đồng ý tới Hoàn toàn đồng ý. Phần này có thêm một câu hỏi phụ về ý kiến góp ý cho nội dung các tiêu chuẩn và tiêu chí của khung đánh giá NLGD tiếng Anh;

- Phần III: Hướng dẫn đánh giá NLGD tiếng Anh: Nó gồm 25 câu hỏi về nội dung hướng dẫn đánh giá NLGD tiếng Anh, và chúng cũng được sử dụng thang đo năm bậc từ Hoàn toàn không đồng ý tới Hoàn toàn đồng ý. Phần này có thêm một câu hỏi phụ về ý kiến góp ý cho nội dung hướng dẫn đánh giá NLGD tiếng Anh.

\subsection{Qui trình lấy dũ liệu và phân tích dũ liệu}

Để thu thập dữ liệu, nghiên cứu này thực hiện hai giai đoạn lấy dữ liệu. Giai đoạn 1: thu thập dữ liệu về thông tin của SV trước khóa học PPGD tiếng Anh, 129 bảng câu hỏi được phát trực tiếp cho $\mathrm{SV}$ trong buổi đầu tiên của môn học PPGD tiếng Anh 1 và $\mathrm{SV}$ mất trung bình từ 10-14 phút để hoàn thành bảng câu hỏi. Tuy nhiên, chỉ có 93 bảng câu hỏi được gởi lại và hợp lệ. Giai đoạn 2: thu thập thông tin đánh giá khung đánh giá NLGD tiếng Anh từ chuyên gia, ba phiếu đánh giá khung NLGD tiếng Anh kèm theo khung đánh giá NLGD tiếng Anh hoàn chỉnh 
được gởi trực tiếp cho ba chuyên gia. Thông tin đánh giá được gởi lại cho nhà nghiên cứu sau khi được hoàn thành sau một tuần.

Để phân tích dữ liệu, phần mềm SPSS được sử dụng để phân tích dữ liệu định lượng và phương pháp phân tích nội dung được sử dụng để phân tích dữ liệu định tính.

\section{Kết quả và bàn luận}

\subsection{Kết quả}

Phần này trình bày kết quả của ba bước (Analyze: phân tích; Design: thiết kế; Develop: phát triển) khung đánh giá NLGD tiếng Anh.

\section{Bước 1: Phân tích (Analyze)}

\section{Xác định nhu cầu và mục đỉch}

Khoa tiếng Anh tại nơi nghiên cứu có thêm chuyên ngành PPGD tiếng Anh với tổng số lượng SV đăng ký học là 129 SV. Mục tiêu đào tạo của chuyên ngành PPGD tiếng Anh, ngoài các mục tiêu chung, là giúp SV có khả năng vận dụng kiến thức về giáo học pháp trọng hoạt động giảng dạy, kiểm tra đánh giá và quản lý các lớp học tiếng Anh. Tuy nhiên, việc đánh giá NLGD tiếng Anh của SV để đảm bảo chất lượng chuẩn đầu ra của ngành PPGD tiếng Anh còn chưa có. Do đó, nhất thiết phải có một khung đánh giá NLGD tiếng Anh nhằm mục đích:

- giúp cho việc đào tạo đảm bảo đúng với chuẩn đầu ra của chuyên ngành PPGD tiếng Anh;

- giúp cho giảng viên có thể định hướng phát triển NLGD tiếng Anh của SV một cách khoa học và đồng bộ hơn;

- giúp cho SV chuyên ngành PPGD tiếng Anh có thể tự định hướng phát triển NLGD tiếng Anh của họ để có thể đáp ứng chuẩn nghề nghiệp.

\section{Phân tích môi trường học}

Kết quả của phân tích môi trường học tương đồng với nội dung trình bày mục 3.2 Nơi nghiên cứu.

\section{Phân tích đặc điểm người học}

Ngoài các thông tin cá nhân đã được trình bày ở mục 3.2 (Đối tượng khảo sát), thông tin ở Bảng 3 cho thấy trước khoa học, SV có thái độ về khóa học PPGD tiếng Anh tích cực $(\mathrm{M}=3,98 ; \mathrm{SD}=, 55)$, nhưng kiến thức $(\mathrm{M}=2,97 ; \mathrm{SD}=, 87)$ và kỹ năng $\mathrm{PPGD}$ tiếng $\mathrm{Anh}(\mathrm{M}=3,00$; $\mathrm{SD}=, 87$ ) ở mức độ không có ý kiến. Điều này có thể hiểu rằng, $\mathrm{SV}$ đã định hướng chọn nghề nghiệp giảng dạy tiếng Anh là nghề nghiệp tương lai nên họ có thái độ tích cực đối với khóa học PPGD tiếng Anh, nhưng đây là khóa học mới nên kiến thức và kỹ năng về PPGD tiếng Anh còn chưa xác định được. 


\section{Bảng 3}

NLGD của SV trước khóa học

\begin{tabular}{|l|c|c|c|}
\hline \multirow{2}{*}{ NLGD tiếng Anh trước khóa học } & \multicolumn{3}{|c|}{ n=93 } \\
\cline { 2 - 4 } & Thái độ M(SD) & $\begin{array}{c}\text { Kiến thức } \\
\mathrm{M}(\mathrm{SD})\end{array}$ & Kỹ năng M(SD) \\
\cline { 2 - 4 } & $3,98(, 55)$ & $2,97(, 87)$ & $3,00(, 87)$ \\
\hline
\end{tabular}

Trong đó: n: số lượng mẫu; $\mathrm{M}$ (mean): trung bình; SD (standard deviation): Độ lệch chuẩn

Nguồn: Kết quả phân tích dữ liệu của nhóm nghiên cứu

Ngoài ra, về năng lực ngôn ngữ Anh thì có thể nhận xét rằng $\mathrm{SV}$ có đủ năng lực để theo học khóa học PPGD tiếng Anh vì họ đang học năm thứ 4 và đã hoàn thành xong các môn học kỹ năng ngôn ngữ bắt buộc theo khung chương trình học.

Tóm lại, trước khi học các môn về PPGD tiếng Anh, mặc dù SV có thái độ rất tích cực về các môn GDTA và có đủ năng lực ngôn ngữ Anh để sử dụng giảng dạy, nhưng họ còn thiếu kiến thức và kỹ năng về PPGD tiếng Anh.

\section{Bước 2: Thiết kế (Design)}

\section{Xác định muc tiêu đánh giá}

Trên cơ sở mục tiêu đào tạo của chuyên ngành PPGD tiếng Anh, mục tiêu của khung đánh giá NLGD tiếng Anh như sau:

1. Thái độ: có thái độ tích cực đối với chuyên ngành PPGD tiếng Anh và các môn học của chuyên ngành PPGD tiếng Anh;

2. Kiến thức: có kiến thức về lý thuyết về quá trình học ngôn ngữ, người học và người dạy; thiết kế bài giảng; sử dụng tài liệu giảng dạy và tài nguyên giảng dạy; quản lý lớp học, và xử lý các tình huống sư phạm; nguyên lý và kỹ thuật giảng dạy; kiểm tra đánh giá người học; sử dụng CNTT và đồ dùng dạy học trong giảng dạy; và dự giờ;

3. $K \tilde{y}$ năng: có kỹ năng ứng dụng lý thuyết về quá trình học ngôn ngữ, người học và người dạy vào giảng dạy; thiết kế bài giảng; sử dụng tài liệu giảng dạy và tài nguyên giảng dạy; quản lý lớp học, và xử lý các tình huống sư phạm; giảng dạy các kỹ năng; thiết kế kiểm tra đánh giá người học; sử dụng CNTT và đồ dùng dạy học trong giảng dạy; và ghi chép dự giờ;

4. Năng lục ngôn ngũu: Có đủ năng lực về ngôn ngữ tiếng Anh bậc 5 theo khung năng lực ngoại ngữ Việt Nam.

\section{Xác định nội dung đánh giá}

Trên cơ sở của mục tiêu đánh giá, nội dung đánh giá của khung đánh giá NLGD tiếng Anh được thể hiện ở 9 tiêu chuẩn sau:

1. Quá trình học ngôn ngũu, nguời dạy và nguời học: Có kiến thức về các lý thuyết về quá trình học ngôn ngữ, người dạy và người học và kỹ năng để áp dụng các lý thuyết đó vào ngữ cảnh giảng dạy cụ thể; 
2. Thiết kế bài dạy: Có kiến thức và kỹ năng về tiến trình soạn giảng 1 bài học cụ thể;

3. Sủ dụng tài liệu và đồ dùng dạy học: Có kiến thức và kỹ năng về đánh giá, lựa chọn, sửa đổi tài liệu và sử dụng dạy học phù hợp với ngữ cảnh giảng dạy;

4. Quản lý lớp học và xủ lý tình huống sur phạm: Có kiến thức và kỹ năng về quản lý lớp học và sử dụng ngôn ngữ trong lớp và xử lý tình huống sư phạm một cách mô phạm;

5. Kỹ năng giảng dạy: Có kiến thức và kỹ năng giảng dạy về từ vựng, ngữ pháp, phát âm, nghe, nói, đọc, và viết;

6. Đánh giá người học: có kiến thức và kỹ năng về đánh giá người học theo các hình thức khác nhau;

7. Úng dụng CNTT và đồ dùng dạy học: Có kiến thức và kỹ năng về ứng dụng CNTT và đồ dùng dạy học vào các bài giảng cụ thể;

8. Dư giơ: Có kiến thức và kỹ năng về ghi chép và đánh giá 1 tiết dạy học cụ thể;

9. Năng lực ngôn ngũu: Có đủ năng lực về ngôn ngữ tiếng Anh bậc 5 theo khung năng lực ngoại ngữ Việt Nam.

Việc đánh giá NLGD tiếng Anh của SV thành 2 cấp (không đạt và đạt) với 5 mức độ như sau:

\section{- KHÔNG ĐẠT}

1. Kém: NLGD của SV ở mức KÉM khi điểm đánh giá của các tiêu chuẩn trong NLGD tiếng Anh từ 0.0 đến 3.9 điểm tính theo thang điểm 10 .

\section{- ĐẠT}

2. Trung bình-Yếu: NLGD của SV ở mức TRUNG BÌNH-YẾU khi điểm đánh giá của các tiêu chuẩn trong NLGD tiếng Anh từ 4.0 đến 5.4 điểm tính theo thang điểm 10 ;

3. Trung bình: NLGD của SV ở mức TRUNG BÌNH khi điểm đánh giá của các tiêu chuẩn trong NLGD tiếng Anh từ 5.5 đến 6.9 điểm tính theo thang điểm 10;

4. Khá: NLGD của SV ở mức KHÁ khi điểm đánh giá của các tiêu chuẩn trong NLGD TA từ 7.0 đến 8.4 điểm tính theo thang điểm 10 ;

5. Giỏi: NLGD của SV ở mức GIỎI khi điểm đánh giá của các tiêu chuẩn trong NLGD tiếng Anh từ 8.5 đến 10.0 điểm tính theo thang điểm 10 .

\section{Bước 3: Phát triển (Develop)}

\section{Phát triển khung đánh giá}

Dựa trên các bước đã được triển khai trước, khung đánh giá NLGD tiếng Anh được phát triển hướng dẫn chi tiết thực hiện đánh giá được phát triển. Khung đánh giá NLGD tiếng Anh gồm ba phần: 
- Phần A: Mục tiêu của khung đánh giá NLGD tiếng Anh. Phần này trình bày bốn mục tiêu và 9 tiêu chuẩn của khung đánh giá NLGD tiếng Anh;

- Phần B: Nội dung chi tiết của khung đánh giá NLGD tiếng Anh. Phần này trình bày 24 tiêu chuẩn sắp xếp theo chín tiêu chuẩn của khung đánh giá NLGD tiếng Anh;

- Phần $\mathrm{C}$ : Hướng dẫn đánh giá NLGD tiếng Anh. Phần này trình bày nội dung chi tiết của các 24 tiêu chí được xếp theo chín tiêu chuẩn của khung đánh giá NLGD tiếng Anh.

\section{Thẩm định}

Theo kết quả Bảng 4, chỉ số trung bình của "Mục tiêu của khung đánh giá NLGD tiếng Anh" (Nhóm A: $M=4,35 ; \mathrm{SD}=, 56)$, "Nội dung chi tiết của khung đánh giá NLGD tiếng Anh" (Nhóm $\mathrm{B}: \mathrm{M}=4,41 ; \mathrm{SD}=, 52$ ) và "Hướng dẫn đánh giá NLGD tiếng Anh" (Nhóm $\mathrm{C}: \mathrm{M}=4,35$; $\mathrm{SD}=, 56)$ đều ở mức Hoàn toàn đồng $y ́(4,21<\mathrm{M}<5,00)$. Kết quả này có thể hiểu là các nhà đánh giá hoàn toàn đồng ý với nội dung của khung đánh giá NLGD tiếng Anh.

\section{Bảng 4}

Đánh giá chung về khung đánh giá NLGD tiếng Anh

\begin{tabular}{|c|l|c|c|}
\hline \multirow{2}{*}{ Stt } & \multicolumn{1}{|c|}{ Nội dung } & \multicolumn{2}{|c|}{$\mathbf{n = 3}$} \\
\cline { 3 - 4 } & \multicolumn{1}{|c|}{ M } & SD \\
\hline A & Mục tiêu của của khung đánh giá NLGD tiếng Anh (16 câu) & 4,35 &, 56 \\
\hline B & Nội dung chi tiết của khung đánh giá NLGD tiếng Anh (33 câu) & 4,41 &, 52 \\
\hline C & Hướng dẫn đánh giá NLGD tiếng Anh (25 câu) & 4,35 &, 56 \\
\hline
\end{tabular}

Trong đó: $\mathrm{n}$ : số lượng mẫu; $\mathrm{M}$ (mean): trung bình; $\mathrm{SD}$ (standard deviation): Độ lệch chuẩn

Nguồn: Kết quả phân tích dữ liệu của nhóm nghiên cứu

Đối với câu hỏi mở, có 2 nhà nghiên cứu góp ý bổ sung như sau:

- Phần A: Nội dung mục tiêu về thái độ;

- Phần B: Nội dung các tiêu chí liên quan đến mục tiêu về thái độ;

- Phần C: Nội dung hướng dẫn đánh giá NLGD tiếng Anh của các tiêu chí liên quan đến mục tiêu về thái độ.

Tóm lại, khung đánh giá NLGD tiếng Anh được xây dựng dựa vào nhu cầu thực tế và được thẩm định. Khung đánh giá NLGD tiếng Anh được điều chỉnh dựa trên các bước Xác định nhu cầu và mục đích, Xác định mục tiêu đánh giá, và Xác định nội dung đánh giá.

\section{8. Điều chinh}

Thông tin đánh giá thu thập được từ thẩm định khung đánh giá NLGD tiếng Anh được dùng để điều chỉnh nội dung của các bước Xác định mục tiêu đánh giá, Xác định nội dung đánh giá, và Phát triển khung đánh giá, Tuy nhiên, chỉ có thông tin ở khung đánh giá NLGD tiếng Anh được điều chỉnh như sau:

- Phần A: Mục tiêu của khung đánh giá NLGD tiếng Anh. Nội dung mục tiêu về thái độ được điều chỉnh, và nội dung của chín tiêu chuẩn cũng được điều chỉnh bổ sung phần thái độ; 
- Phần B: Nội dung chi tiết của khung đánh giá NLGD tiếng Anh. Nội dung của 24 tiêu chí được bổ sung.

Kết quả của bước này là khung đánh giá NLGD của SV chuyên ngành PPGD tiếng Anh hoàn chỉnh.

\subsection{Bàn luận}

Kết quả về NLGD tiếng Anh trước khóa học có áp dụng khung đánh giá NLGD tiếng Anh cho thấy $\mathrm{SV}$ có thái độ tích cực $(\mathrm{M}=3,98 ; \mathrm{SD}=, 55)$ đối với khóa học PPGD tiếng Anh. Một trong những lý do để lý giải điều này có thể là do đam mê giảng dạy của họ. Có tới hơn $50 \%$ $(55,9 \%$ - $55 \mathrm{SV})$ trong tổng số $93 \mathrm{SV}$ tham gia khảo sát đã trả lời họ chọn học chuyên ngành PPGD tiếng Anh vì đam mê. Ngoài ra, cũng có cùng tỉ lệ phần trăm đó $(55,9 \%$ - $55 \mathrm{SV})$ về số lượng SV trả lời rằng họ đã có kinh nghiệm giảng dạy với các vị trí như trợ giảng, gia sư và thậm chí là $\mathrm{GV}$ chính thức tại các cơ sở giảng dạy tiếng Anh. Chính vì lý do đó đã giúp cho $\mathrm{SV}$ có thái độ tích cực đối với khóa học PPGD tiếng Anh.

Tuy nhiên, về khía cạnh kiến thức $(\mathrm{M}=2,97 ; \mathrm{SD}=, 87)$ và kỹ năng về PPGD tiếng Anh $(\mathrm{M}=3,00 ; \mathrm{SD}=, 87)$ của $\mathrm{SV}$ không có ý kiến. Điều này cũng dễ hiểu vì nội dung kiến thức và kỹ năng về giảng dạy PPGD tiếng Anh là mới đối với họ nên họ không chắc là có kiến thức và kỹ năng về giảng dạy hay không dù hơn $50 \%$ trong số $93 \mathrm{SV}$ đã có kinh nghiệm thực hiện giảng dạy ở các vị trí khác nhau. Ngoài ra, nhiều kiến thức và kỹ năng liên quan đến cách soạn giáo án, cách đánh giá người học, tổ chức hoạt động học tập, quản lý lớp học, cách ghi chép dự giờ, đánh giá giờ dạy, sử dụng CNTT trong giảng dạy tiếng Anh, và soạn đề kiểm tra đánh giá người học đều mới với $\mathrm{SV}$.

\section{Kết luận}

Khung đánh giá NLGD tiếng Anh được xây dựng dựa trên theo mô hình ADDIE khép kín theo hệ thống nhằm đáp ứng nhu cầu thực tế, nên nó có thể mang tính hữu dụng cao cho chương trình đào tạo chuyên ngành PPGD tiếng Anh tại nơi nghiên cứu. Tuy nhiên, trong phạm vi bài nghiên cứu này, khung đánh giá NLGD tiếng Anh chưa được đánh giá qua thực tiễn để xác định tính hiệu quả của khung này. Ngoài ra, hạn chế của nghiên cứu này là nội dung các tiêu chí của khung đánh giá NLGD tiếng Anh chưa được thiết kế tương thích với các tiêu chuẩn và tiêu chí của CDIO (Conceive - Design - Implement - Operate: Hình thành ý tưởng, thiết kế ý tưởng, thực hiện và vận hành) hay AUN-QA (Mạng lưới Đảm bảo chất lượng của hệ thống các trường đại học ASEAN: ASEAN University Network - Quality Assurance). Do đó, nghiên cứu tiếp theo nên tìm hiểu tính hiệu quả của khung đánh giá NLGD tiếng Anh khi được áp dụng vào đánh giá thực tế trong môn thực hành giảng dạy để xem NLGD tiếng Anh của SV phát triển như thế nào.

\section{Tài liệu tham khảo}

Alqiawi, D. A., \& Ezzeldin, S. M. (2015). A suggested model for developing and assessing competence of prospective teachers in faculties of education. World Journal of Education, 5(6), 65-73. doi:10.5430/wje.v5n6p65 
BALEAP. (2008). Competency framework for teachers of English for academic purposes. Retrieved January 15, 2020, from https://bit.ly/37vK1li

Barman, P., \& Paramanik, N. (2019). Status of the teacher competency among the B. Ed., trainee teachers: An analytical study. International Journal of Research in Social Sciences, 9(2), 477-488.

Bhargava, A., \& Pathy, M. (2011). Perception of student teachers about teaching competencies. American International Journal of Contemporary Research, 1, 77-81.

Bộ Giáo Dục và Đào Tạo (BGDĐT). (2014). Khung năng lục ngoại ngũ 6 bậc dùng cho Việt Nam [6-level language proficiency framework for Vietnamese use]. Retrieved January 16, 2020, from shorturl.at/fiV07

Branch, R. M. (2009). Instructional design: The ADDIE approach. New York, NY: Springer.

British Council. (2011). CPD framework for teachers of English. Retrieved January 17, 2020, from https://bit.ly/2qBZwaA

British Council. (2012). Going forward: Continuing professional development for English teachers in the UK. Retrieved January 18, 2020, from https://bit.ly/2pNOP6k

Bui, M. D., Nguyen, N. T., Dao, T. V. A., \& Hoan, T. K. H. (2017). In the development of professional standards for Vietnamese teachers: Studies on Singapore's experience. International Journal of Educational Science and Research (IJESR), 7(2), 149-154.

Center for Adult English Language Acquisition (CAELA) Network. (2010). Framework for quality professional development for practitioners working with adult English language learners. Retrieved January 19, 2020, from https://bit.ly/2XEkC41

Council of Europe. (2008). White paper of intercultural dialogue: Living together as equals in dignity. Strasbourg, France: Council of Europe.

Darling-Hammond, L., \& Branford, J. (2005). Preparing teachers for a changing world: What teachers should learn and be able to do. San Francisco, CA: Jossey-Bass.

Deakin, C. R. (2008). Pedagogy for citizenship. In F. Oser \& W. Veugelers (Eds.), Getting involved: Global citizenship development and sources of moral values, (pp. 31-55). Rotterdam, Netherlands: Sense Publishers.

Duong, T. M., Pham, T. T. H., \& Thai, H. L. T. (2012). Building an assessment competence framework for pre-service and in-service ELT teachers in Vietnam. Paper presented at TESOL Conference, University of Languages and International Studies, Hanoi, Vietnam.

EAQUALS. (2013). The eaquals framework for language teacher training and development. Retrieved January 20, 2020, from http://eaquals,org/pages/7104

Feiman-Nemser, S. (2008). Teacher learning. How do teachers learn to teach? In M. C. Smith, S. Feiman-Nemser, \& D. McIntyre (Eds.), Handbook of research on teacher education, enduring questions in changing contexts. New York, NY: Routledge; Abingdon, France: Taylor \& Francis.

Hagger, H., \& McIntyre, D. (2006). Learning teaching from teachers. Realizing the potential of school-based teacher education. Maidenhead, UK: Open University Press. 
Hatano, G., \& Oura, Y. (2003). Commentary: Reconceptualising school learning using insight from expertise research. Educational Researcher, 32(8), 26-29. doi:10.3102/0013189X032008026

Koster, B., \& Dengerink, J. J. (2008). Professional standards for teacher educators: How to deal with complexity, ownership and function, experiences from the Netherlands. European Journal of Teacher Education, 31(2), 135-149.

Krauss, S., Brunner, M., Kunter, M., Baumert, J., Blum, W., Neubrand, M., \& Jordan, A. (2008). Pedagogical content knowledge and content knowledge of secondary mathematics teachers. Journal of Educational Psychology, 100(3), 716-725.

McDiarmid, W. G., \& Clevenger-Bright, M. (2008). Rethinking teacher capacity. In M. C. Smith, S. Feiman-Nemser, \& D. McIntyre (Eds.) Handbook of research on teacher education, enduring questions in changing contexts. New York, NY: Routledge; Abingdon, France: Taylor \& Francis.

Mishra, P., \& Koehler, M. (2006). Technological pedagogical content knowledge: A framework for teacher knowledge. Teachers College Record, 108, 1017-1054. doi:10.1111/j.14679620.2006.00684.x

Pham, H. A., \& Ta, T. B. (2016). Developing a Theoretical Framework for ESP teacher training in Vietnam. The ESP Journal, 12(1), 66-84.

Rychen, D. S., \& Salganik, L. H. (2003). Key competencies for a successful life and a wellfunctioning society. Göttingen, Germany: Hogrefe \& Huber.

Scheerens, J., Luyten, J., Steen, R., \& Luyten-de Thouars, Y. (2007). Review and metaanalyses of school and teaching effectiveness. Enschede, Netherlands: University of Twente, Department of Educational Organisation and Management.

Shulman, L. S. (1987). Knowledge and teaching: Foundations of the new reform. Harvard Educational Review, 57(1), 1-23. doi:10.17763/haer.57.1.j463w79r56455411

Tran, Q. T. (2020). Student teachers' perception of their teaching competency assessed by a framework for assessing student teachers' English teaching competency (FASTETC). VNU Journal of Foreign Studies, 36(3), 164-177. doi:10.25073/2525-2445/vnufs.4563

UCLES. (2015). Cambridge English teaching framework: How and why the framework was developed. Cambridge, MA: Cambridge English Language Assessment.

Vogt, F., \& Rogalla, M. (2009). Developing adaptive teaching competency through coaching. Teaching and Teacher Education, 25(8), 1051-1060. doi:org/10.1016/j.tate.2009.04.002 